\title{
Measurements of Low-Angle Radiation From a Monopole
}

\author{
Alvin C. Wilson

\begin{abstract}
Contribution from Central Radio Propagation Laboratory, National Bureau of Standards, Boulder, Colo.
\end{abstract}
(Received April 27, 1961; revised June 27, 1961)

\begin{abstract}
Experimental measurements using scale model techniques have been carried out to determine the effectiveness of a ground system of long-wire radials to obtain low angles of departure of transmission. Since transmission was to be in one direction only, the ground wires were laid out to form a ground-plane sector approximately $18^{\circ}$ wide centered in the direction of transmission. The antenna was a base-driven vertical monopole. Measurements were made of the relative response in decibels for the monopole used as a receiving antenna at a frequency of 400 megacycles per second. The target transmitter antenna was always located at a distance of 200 wavelengths. At this separation the ground plane sector was in the near field of the target transmitting antenna and appropriate corrections must be made.

The received signal strength improvement due to the presence of the ground sector was approximately 14 decibels. The measured lobe positions of the first and second beam maximums and the first null are in good agreement with theory.
\end{abstract}

\section{Introduction}

In connection with a $\mathrm{HF}$ arctic propagation project using a base-driven vertical monopole at a frequency of $10 \mathrm{Mc} / \mathrm{s}$ over a ground of lava rock having a poor conductivity, a requirement arose to evaluate the effectiveness of a planned long-wire ground radial system in improving low-angle response.

A common practice has been to use a ground system of wire radials centered at the antenna base and extending outward for a distance of approximately one-half wavelength [Laport, 1952]. However, an improved ground system was desired to obtain low angles of departure of transmission.

One method of obtaining a low angle of departure would be to elevate the base of the antenna above ground. However, this would result in multiple lobes in the vertical plane. It was then believed to be advantageous to improve on the effectiveness of the ground radial system and maintain the antenna base at ground level.

Since the transmission path was in one direction only, a ground system was planned which would consist of wire radials $3,000 \mathrm{~m}$ long (approximately 100 wavelengths) covering an approximate segment of ground $18^{\circ}$ wide in the desired direction of transmission.

Before the start of any actual construction, a study of this ground system of long-wire radials, using modeling techniques, was desired. Measurements were made to obtain the signal response over the center of the ground radial system and at other points over the ground-plane sector compared to the signal response over the ground path only. This would show the effect of the ground-plane sector width, the signal improvement due to the long wire radials, and the improvement in response with different numbers of wire radials (density of wires in the ground-plane sector).

This report presents the results of the measurements which were carried out at a frequency of 400 $\mathrm{Mc} / \mathrm{s}$ using scaled model techniques.

\section{Method of Measurement}

For the purposes of measurement, the ground radial system to be measured was scaled for a frequency of $400 \mathrm{Mc} / \mathrm{s}$ (a scaling factor of 40). The measurements were carried out at the NBS antenna range on Table Mesa near Boulder, Colo. The ground area over which the measurements were carried out was first cleared of weeds, brush, and large rocks in order to make the ground as smooth as possible. The ground conductivity is considered to be poor and no attempt was made to scale the ground conductivity.

A quarter wavelength monopole, extended vertically from the center of a flat aluminum disk having a diameter of one wavelength. This disk was to represent a conventional ground system of a number of wires each one-half wavelength long.

The long-wire ground system initially consisted of 97 ground wires of No. 22 AWG Copperweld, each $75 \mathrm{~m}$ long. These wires covered an approximate $18^{\circ}$ sector. The ends of the wires, separated by $\lambda / 3\left(0.191^{\circ}\right.$ angular separation) were secured in position and bonded to $20 \mathrm{~cm}$-long steel spikes which were driven into the ground until the tops of the spikes were flush with the ground surface. The other ends of the radials were bonded to the edge of the flat aluminum disk.

The vertical monopole was used as a receiving antenna. The target transmitter antenna, a 5- 
element Yagi oriented for vertical polarization, was moved in a $40^{\circ}$ azimuthal arc at a distance of $75 \mathrm{~m}$ in front of the wire radial ends. The whole ground plane sector was not in the far field of the transmitting Yagi antenna. The measurements were made by recording the relative received response in decibels by the monopole. Figure 1 is a plan view of the ground radial system. The response in decibels versus azimuth position of the transmitting antenna was measured at a constant angle of arrival of $1.1^{\circ}$. The response in decibels versus angle of arrival in the range of $0^{\circ}$ to $6.73^{\circ}$ was measured at the sector center position. The angle of arrival is the included angle at the base of the monopole between the horizon and the target transmitter antenna.

The measurements of response in decibels versus azimuth position of the transmitting antenna at a constant angle of arrival of $1.1^{\circ}$ were also carried out for ground systems of 49 wires $\left(0.382^{\circ}\right.$ angular separation) and 25 wires $\left(0.764^{\circ}\right.$ angular separation) by removing every other wire from the original 97 and then remaining 49 wires.

\section{Results}

Figure 2 presents the curves of record ed relative response in decibels versus azimuth position of the transmitting antenna for the ground system consisting of 97,49 , and 25 wires, measured over a $40^{\circ}$ ground sector. The received signal is generally higher when measured at azimuth positions over the grov u- $^{-}$ plane sector center as is to be expected. The received signal is also highest in the case when the ground-plane sector was of maximum density of wie radials (97 wires). The fall-off of the received signal when measured over a path of ground only (no long wire radials) is gradual, and at $20^{\circ}$ each side of the ground-sector center the long wire radials do not appear to have any effect on the received signal strength.

Figure 3 is a plot of the relative response in decibels versus the angle of arrival in the forward direction with a ground-plane sector of 97 wires. Measurements at angles above $6.73^{\circ}$ were not possible because of the maximum height of mounting for the target transmitter antenna and separation distance between the receiving monopole and transmitting antenna.

\section{Discussion of Results}

On the assumption that the wire radials form a well conducting ground plane, and from the geometry of figure 1, computations can be made to compare theoretically computed values [Wait and Conda, 1958a] with the experimental results shown in figure 3 .

The computed position of the first beam maximum is $3.3^{\circ}$. The experimental value of this first maximum is $2.4^{\circ}$. The position of the second maximum is computed to be $6.2^{\circ}$ and the experimental value

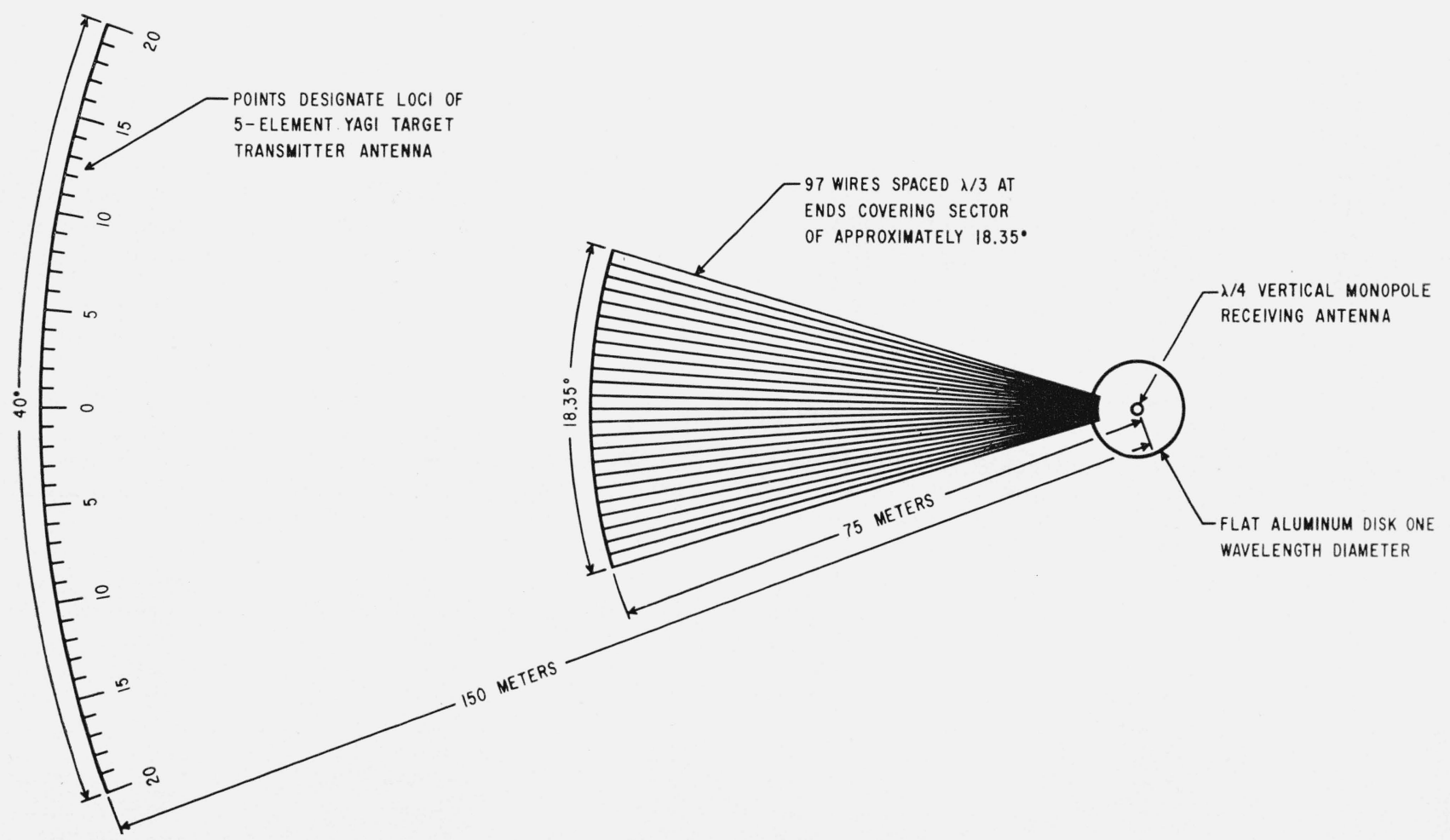

FIGURE 1. Plan view drawing of extended wire radial ground system modeled for measurement at $400 \mathrm{Mc} / \mathrm{s}$. 


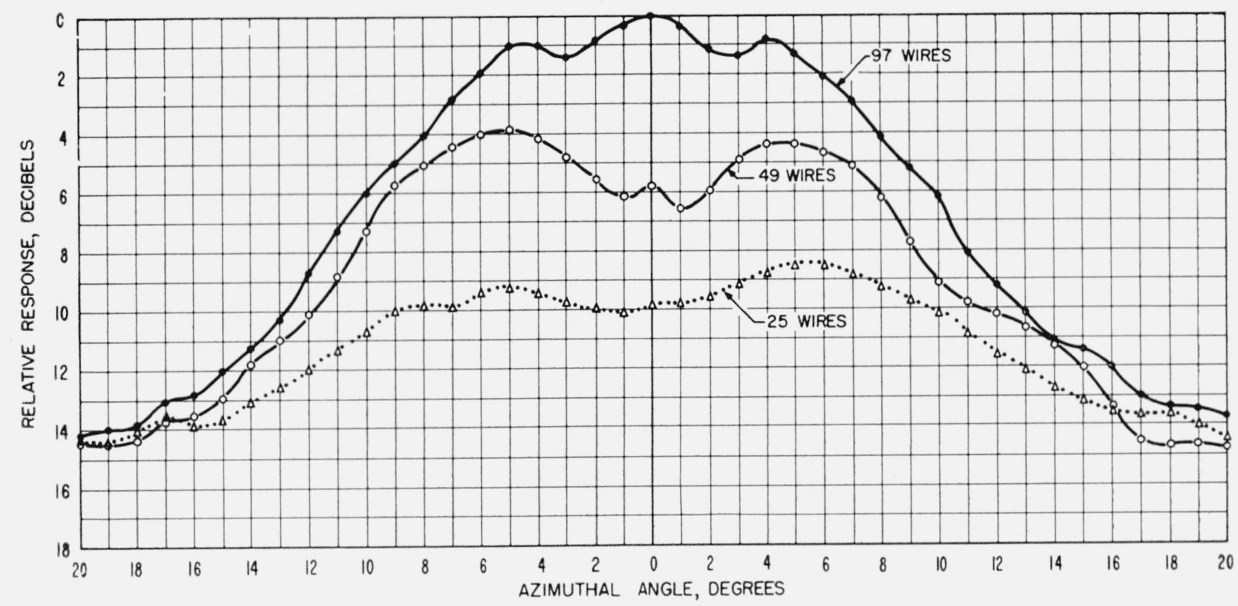

Figure 2. Curves of relative response, decibels, versus azimuthal angle, degrees, for extended wire radial ground systems of 97,49 , and 25 wires.

The angle of signal arrival is 1.10 in all cases.

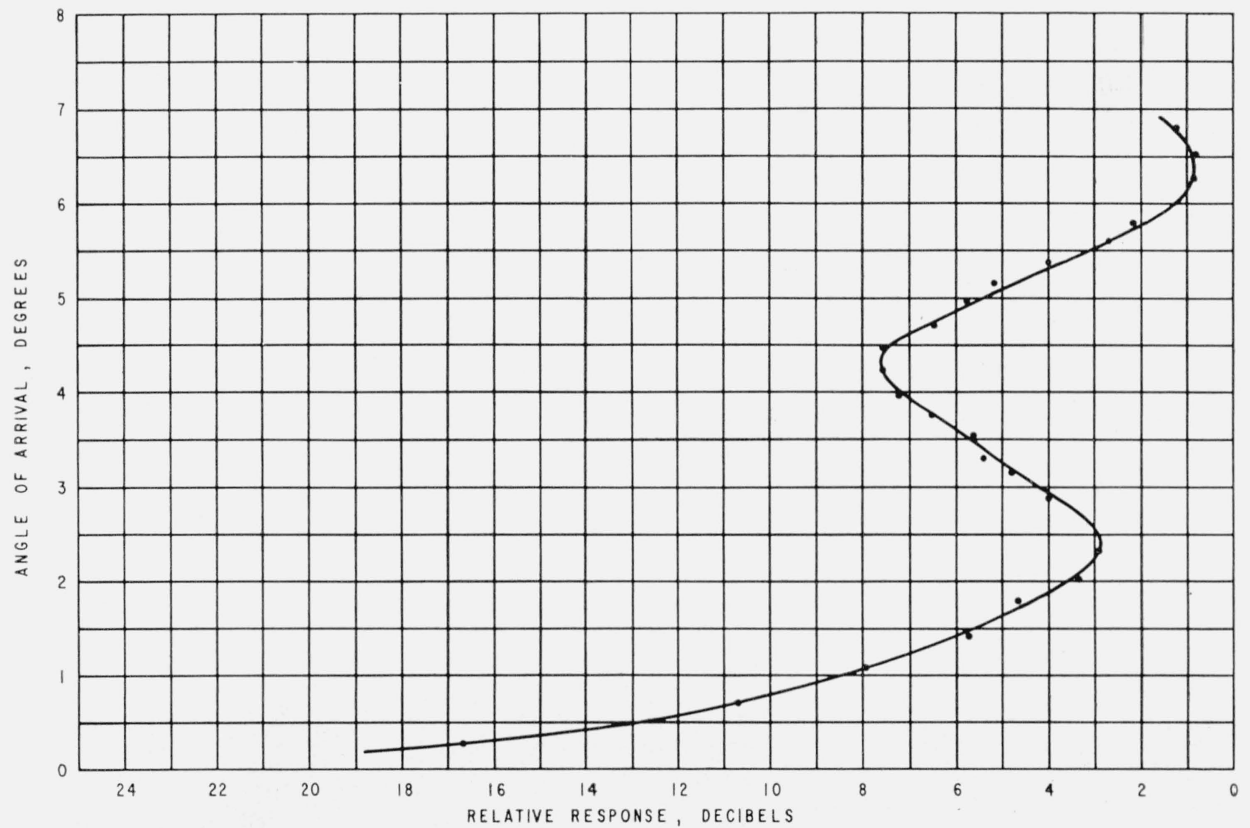

FIGURE 3. Relative response of the monopole in the vertical plane in the forward direction over an extended wire radial system of 9 wires.

is $6.3^{\circ}$. The position of the experimental first null is at an angle of $4.3^{\circ}$. This compares to a computed first null position of $5.0^{\circ}$.

With the exception of the first maximum, these experimental results agree quite well with a simple model of conducting half plane to represent the ground plane. The finite conductivity of the ground outside the ground plane will tend to lower the first lobe maximum [Wait and Conda, 1958a].

While the results of the model measurements are optimistic as regards low angle radiation, special considerations must be made when interpreting the results of the model measurements as related to the HF case. This is necessary since the angle of the lobes (for small departure angles) are approximately proportional to $\sqrt{1-\frac{75}{150}}$ where 75 is the length of the long wire radials and 150 is the horizontal separation between transmitting antenna and the receiving monopole (see fig. 1). If the separation between the transmitting antenna and receiving monopole increases from $150 \mathrm{~m}$ to infinity as in the field case, 
the lobes are raised in elevation. For this experiment $\sqrt{1-\frac{75}{150}}=\frac{1}{2}$. Thus the experimentally measured lobe positions should be raised by more than 40 percent for optimum siting of a point-to-point $\mathrm{HF}$ link.

Another consideration to be made when interpreting the scaled model measurements as regards the $\mathrm{HF}$ case is the influence of the earth curvature. At these low angles the pattern is cut back due to diffraction. For the $10 \mathrm{Mc} / \mathrm{s} \mathrm{HF}$ case this would amount to approximately 1/2 degree [Wait and Conda, 1958b]. Thus some error could be expected due to the earth curvature, particularly at low angles of the order of 1 degree.

\section{Conclusions}

The results of these measurements show that an increase in the density (number of wires in the sector) improves the relative level of the received signal.

Measurements of ground conductivity, effect of increased wire density, and effect of even longer wire radial ground systems were beyond the scope of these measurements. In order to completely investigate the advantages of ground systems with extended wire radials, it would be desirable to make a further study with an additional number of wires and wire lengths, and to carry out measurements at higher angles of arrival.

This work was carried out in 1959 in support of the HF Arctic Propagation Project No. 85421 sponsored by the U.S.N. BuShips.

The measurements were made with the assistance of W. L. Martin, G. W. Angus, and R. W. Harsh, of the National Bureau of Standards, and W. E. McKenney and F. A. Foss of Scatter Communication Corporation.

\section{References}

Laport, E. A., Radio Antenna Eng., pp. 82 and 117 (McGrawHill Book Co. Inc., New York, N.Y., 1952).

Wait, J. R. and A. M. Conda, The patterns of a slot-array on a finite ground plane, L'Onde Electrique Special Supplement, Vol. 1, pp. 21-29, Proc. of the International Congress on UHF Circuits and Antennas, Paris (1958a).

Wait, J. R., and A. M. Conda, Pattern of an antenna on a curved lossy surface, IRE Trans, on Antennas and Prop. AP-6, No. 4, 348-359 (Oct. 1958b).

\section{Appendix ${ }^{1}$}

\subsection{Path Geometry}

A very important consideration for evaluating measurements as described in this paper is that the target transmitter is at a finite distance whereas the actual source of the field for a full size antenna is a downcoming plane wave (or at least something approaching it). Figure 4 shows the geometry of the path.

\footnotetext{
1 This appendix is based on a private communication from J. R. Wait.
}

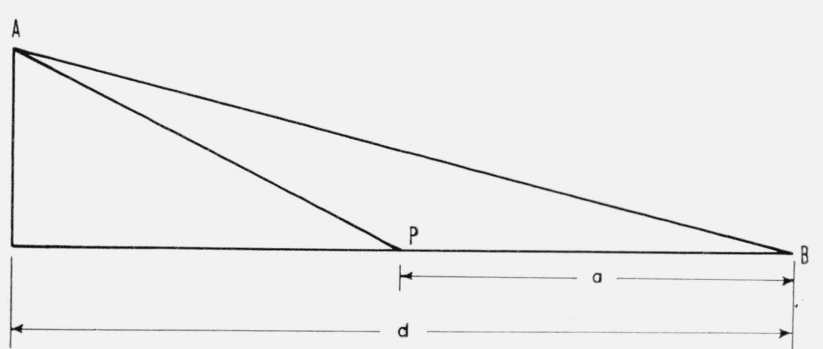

Figure 4. Drawing of measurement path geometry. Transmitting antenna at point A; receiving monopole at point B.

On the assumption that PB is a well conducting ground plane it can be expected that the antenna pattern first maximum in the vertical plane occurs when $\Delta=A P+P B-A B=m^{2} \frac{\lambda}{4}$ when $m^{2}$ is some number of order unity.

By trigonometry the following will be obtained:

$$
\Delta=\left[(d \tan \psi)^{2}+(d-a)^{2}\right]^{1 / 2}+a-\frac{d}{\cos \psi},
$$

If $\psi$ is small this simplifies to

$$
\Delta \simeq \frac{a \psi^{2}}{2}\left[\frac{1}{1-\frac{a}{d}}\right]
$$

The angle $\psi$ which satisfies $\Delta=m \frac{\lambda}{4}$ is

$$
\psi_{0} \simeq m\left\{\frac{\lambda}{2 a}\left[1-\frac{a}{d}\right]\right\}^{1 / 2}
$$

The value of $m$ can be identified with the value of $u$ where the function

$$
F(u)=\frac{1+i}{2}\left\{\left[\frac{1}{2}+C(u)\right]-i\left[\frac{1}{2}+S(u)\right]\right\} .
$$

is a maximum. $C$ and $S$ are Fresnel integrals [Wait and Conda, 1958a]. Thus $m \simeq 1.2$.

For the measurements carried out at a frequency of $400 \mathrm{Mc} / \mathrm{s}$ the first maximum is obtained. $\quad a=75 \mathrm{~m}$ thus $\frac{2 a}{\lambda} \simeq 14$ and

$$
\begin{aligned}
\psi_{0} & \simeq \frac{m}{14}\left(1-\frac{a}{d}\right)^{1 / 2} \text { radians } \\
& \simeq 4 m\left(1-\frac{a}{d}\right)^{1 / 2} \text { degrees } \\
\psi_{0} & \simeq 2.8 m \simeq 3.3^{\circ}
\end{aligned}
$$

For the second maximum $m \simeq 2.2$ which gives $\psi_{0} \simeq 6.2^{\circ}$. For the first null $m \simeq 1.8$ which gives $\psi_{0} \simeq 5.0^{\circ}$. 


\subsection{Earth Curvature Influence}

Another consideration in evaluating the described measurements as pertained to an actual operational antenna is the influence of the earth's curvature [Wait and Conda, 1958b].

At these low angles the pattern is "cut back" due to diffraction. The general restriction on neglecting this effect is then

$$
\psi>>\left(\frac{2}{k a}\right)^{1 / 3} \text { radians }
$$

where $k a$ is the circumference of the earth in wavelengths. For $10 \mathrm{Mc} / \mathrm{s}$ this works out to be $\psi \gg 1 / 2$ degree. Thus some error could be expected, particularly at low angles of the order of 1 degree.

(Paper 65D6-167) 